\title{
Rheological properties and small-angle X-ray scattering studies of phosphate dust obtained from baghouse collectors
}

https://doi.org/10.1515/arh-2019-0017

Received Mar 08, 2019; accepted Nov 09, 2019

Abstract: Baghouse dust collectors are using in the drying unit of Beni Idir situated in Beni-Idir Khouribga city, Morocco, to retrieve phosphates particles from dust air-drying before its expulsion through the smokestacks. The phosphate dust samples used in this study were taken from the filtration chamber of the baghouse dust collectors. The first sample (S1) is untreated calcium phosphate dust, the second (S2) is the calcium phosphate dust from the outside of filter media while the third one (S3) is the calcium phosphate dust from the inside of filter media which causes clogging depth. In this paper, the rheology and the smallangle X-ray scattering (SAXS) of the three samples were investigated to elucidate the changes in terms of local structure, the viscosity, and the shear stress parameters. The rheological behavior of the dust samples was investigated for a solid mass concentration ranging from 50 to $60 \%$, the three samples (S1) (S2) and (S3) had a solid mass concentration of $\mathrm{C} 1=60 \%, \mathrm{C} 2=55 \%$ and $\mathrm{C} 3=50 \%$ and a shear rate in the range from 1 to $1000 \mathrm{~s}^{-1}$. The results indicated that during the filtration process, the pseudo-plastic behavior of the dust phosphate changed to that of Bingham. Comparing the results of the sample's viscosity, we found that the viscosity decreased during the process filtration. The SAXS results suggested that the dust phosphate samples possess a fractal surface structure of enormous dust particles with a rough surface interface. This new study highlights the rheological behavior of grain phosphates that could be extrapolated to other mining powder as grains material or in solution. It is important to understand the

\footnotetext{
*Corresponding Author: Khalil El-Hami: University of Sultan Moulay Slimane, Polydisciplinary Faculty of Khouribga, Laboratory of Nanosciences and Modeling, Morocco;

Email: elhami_k@yahoo.com; Tel: (212) 6.73.63.84.24

Ikram Labtaini: University of Sultan Moulay Slimane, Polydisciplinary Faculty of Khouribga, Laboratory of Nanosciences and Modeling, Morocco; Email: ikram.labtaini@gmail.com
}

rheological characteristics of materials, their flow, and the subsequent deformation of matter as a result of the flow.

Keywords: Phosphate dust, rheological properties, shear, viscosity, SAXS measurements, Porod law.

PACS: 83.80.Iz, 66.20.-d

\section{Introduction}

The protection of the environment is a major concern for all industries whose OCP is part. In this light, OCP group had opted for the dust collectors with sleeves; to comply with the standards of dust emissions into the atmosphere and to respect the workplace exposure limits values for dust. An adequate dust phosphate collection systems need to take into consideration not only the sleeve properties but also the attributes of the phosphate dust capturing system [1]. In defining the phosphate dust characteristics as an influencing factor in the performance of the collector system, a number of concerns must be studied. These incorporate rheological properties and the local structure deduced from small angle X-ray scattering. However, several authors [2-6] have worked on the mechanical properties of calcium phosphate given the need for a good recognition of its rheological behavior. So far, different studies have indicated the importance of dust rheological study in the mineral processing unit operations [7]. As in X-ray crystallography, SAXS descends structural information from the interaction of the three-dimensional distribution of electrons in a sample with X-rays. Besides, most structural information about phosphate materials has been achieved using scanning electron microscopy (SEM) and X-ray diffraction. A few studies have employed other experimental techniques such as the SAXS measurements on phosphates powder [8-10].

The filtration process is joined by the engendering of particle puffs emitted during pneumatic cleaning of the baghouse phosphate dust collector. A little residue of phosphate dust flows within the filter media and produces the

¿ Open Access. (c) 2019 I. Labtaini and K. El-Hami, published by De Gruyter. 
clogging depth. For this purpose, the well understanding of the calcium phosphate dust rheological properties is fundamental. Consequently, in the interest of improving the filtration performance and optimizing the pulsejet cleaning during the drying operation, we have for the first time, made a detailed study of the rheological properties and the local structure by SAXS analysis of the phosphate dust samples obtained during drying process. In this paper, the rheological properties such as shear stress and viscosity of calcium phosphate dust as a function of the solid concentrations were investigated. Results revealed the non-Newtonian behavior of the phosphate dust obtained during filtration/cleaning stages. Using SAXS measurements, the focus is on the comparison between the three phosphate dust microstructure.

\section{Method and materiel}

\subsection{Phosphate dust samples}

The three phosphate dust samples are obtained from the baghouse dust collectors located at drying unit of BeniIdir, Khouribga, Morocco. The first sample (S1) is taken from the conveyor that feeds the drying circuit, it is an untreated phosphate dust that came from the central zone area with medium grade and $68.5 \%$ of Bone phosphate lime, the samples (S2) and (S3) are collected from the outside and the inside respectively of the filter bag enclosed by the filtration chamber, they are a medium grade washed and dried phosphates that came from Sidi Chennane zone with $70.7 \%$ of bone phosphate lime. The two samples (S1) and (S2) have undergone a dry sieving to eliminate the particles with a diameter greater than $1.8 \mathrm{~mm}$.

\subsection{Rheological characterization}

The analysis of the phosphate dust rheological properties was completed with a rotary viscometer (Anton-Paar), which is associated with a computer that enclosing the rheological data processing software named (Rhéoplus). The rotor is screwed onto the spindle and the sample is poured into the cup. The Couette geometry system used. For each sample, about $30 \mathrm{~g}$ of phosphate powder was mixed at a different solid mass concentration with water into the following solid mass concentration $60 \%, 55 \%$ and $50 \%$ for the samples (S1) (S2) and (S3) respectively. Rotation varies from high to low shear rate. The rheological measurements were completed at room temperature of $25^{\circ} \mathrm{C}$. The experi- mental conditions were: the shear rate varied from $1 \mathrm{~s}^{-1}$ to $1000 \mathrm{~s}^{-1}$, it was a descending ramp test. The mass concentrations in solids range from $50 \%$ to $60 \%$. the samples were held under each shear rate for 150 seconds. The shear stress - shear rate plots were obtained from the data acquired by the computer.

\subsection{Small-Angle X-ray Scattering}

The SAXS experiments on the three powder samples were performed $100 \mathrm{~s}$ on tape background on the Super SAXS instrument (Bruker-AXS GmBH, Germany) at Aarhus University Denmark, equipped with an optimized NanoSTAR small-angle X-ray scattering camera. The details of the equipment optimization and experimental method are described previously [11]. Briefly, the $\mathrm{Cu} \mathrm{K} \alpha \mathrm{X}$-ray generator is a rotary anode operating at $45 \mathrm{kV}$ with a size of $0.3 \times 0.3$ $\mathrm{mm}$. The X-ray beam was fixed with tow Göbel mirrors and a three-pinhole collimation system. Spectra are recorded with a gas detector (HiSTAR, Bruker AXS) giving an effective q-range of $0.01-0.38 \AA^{-1}$.

\section{Results and discussion}

\subsection{Rheology}

Figure 1 is a plot of the three dust phosphate samples rheology. Figure 1 shows shear stress versus shear rate curves for non-Newtonian dust phosphate slurries. Figure 1 (S1) con-

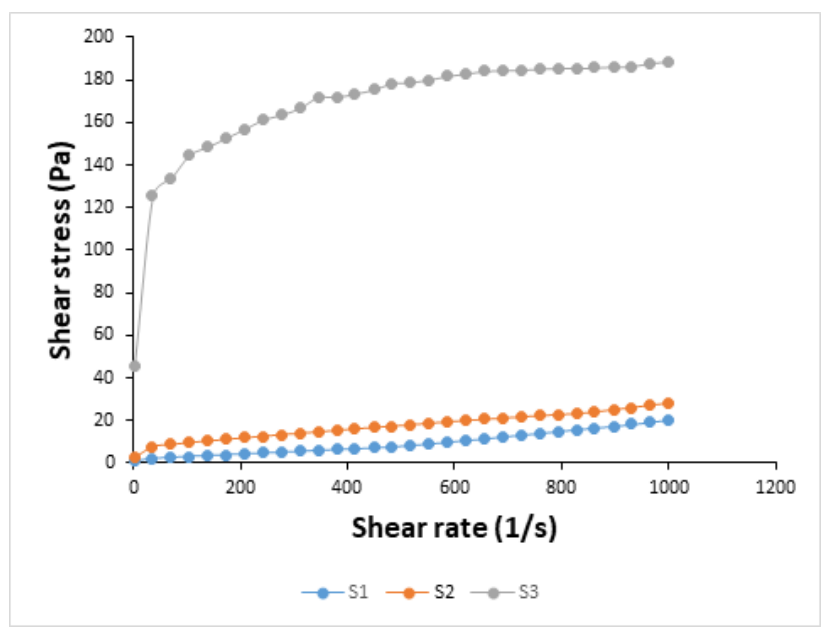

Figure 1: Shear stress dust as a function of rate of shear (sample S1: untreated phosphate dust, sample S2: calcium phosphate dust from the outside of filter and sample S3: calcium phosphate dust from the inside of filter) 


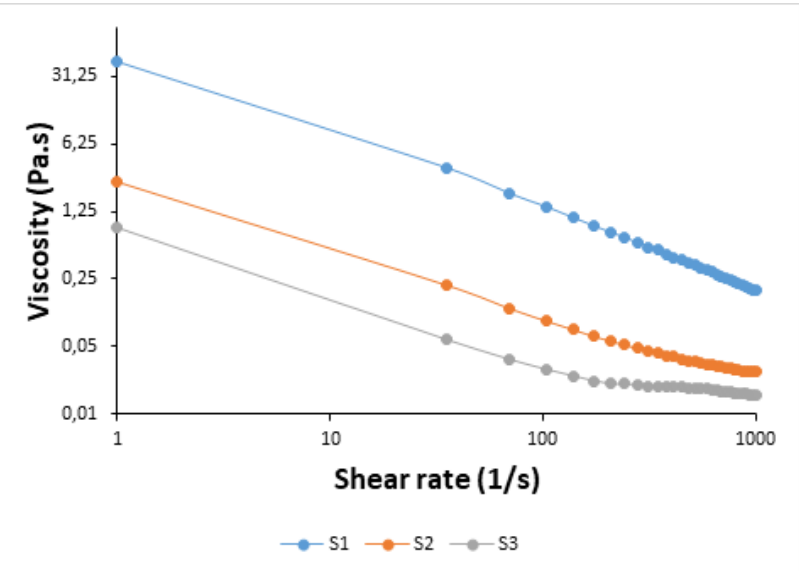

Figure 2: The effect of rate of shear on the dust viscosity (log-log scale) (sample S1: untreated phosphate dust, sample S2: calcium phosphate dust from the outside of filter and sample S3: calcium phosphate dust from the inside of filter)

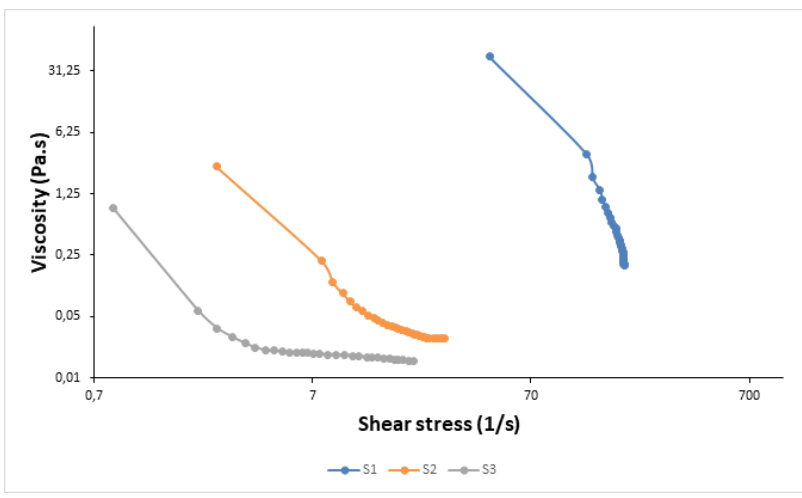

Figure 3: Viscosity versus shear stress (log-log scale) (sample S1: untreated phosphate dust, sample S2: calcium phosphate dust from the outside of filter and sample S3: calcium phosphate dust from the inside of filter)

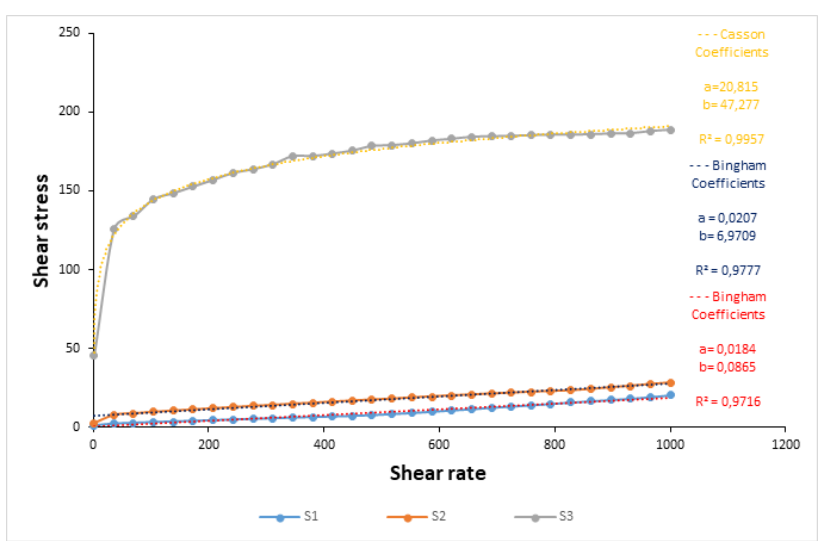

Figure 4: Model fitting (sample S1: untreated phosphate dust, sample S2: calcium phosphate dust from the outside of filter and sample S3: calcium phosphate dust from the inside of filter) tains the variation of the shear stress with the rate of shear at a solid mass concentration of $60 \%$, the time per measuring point is 5 seconds. Untreated dust phosphate showed a pseudo-plastic behavior. Shear stress increased with increasing shear rates for the sample S1, which was typical of pseudo-plastic. Figure 1 (S2 and S3) illustrates the rheological behavior curves of shear stress to a shear rate of the two samples $\mathrm{S} 2$ and $\mathrm{S} 3$. The mass solid concentrations of the samples S2 and S3 were of 55\% 50\%, respectively. The data plots show Bingham behavior. It is observed that the dust phosphate particles change their behavior from pseudo-plastic to Bingham during the filtration process. This is maybe explained by the agglomerates containing in the samples S2 and S3, the agglomerates are created because of the moisture content in the samples which is $2 \%$. Figure 2 shows the effect of rate of shear on the dust viscosity. The viscosity of the dust phosphate samples showed similar behavior. The viscosity decreased rapidly with initial increases in shear rate particularly for the untreated dust phosphate (sample (S1)) indicating that the dust phosphate was a non-Newtonian fluid showing shear thinning. For the three samples, the viscosity diminishes and leans to a minimum at a shear rate of $1000 \mathrm{~s}^{-1}$. The changes in viscosity values of different samples are related to different 'particle interactions' under shear [12]. Figure 3 represents the viscosity versus shear stress of the three samples, as regards the fact that in terms of many applications the dependence of flow on shear stress is more suitable-we tend now to plot viscosity vs shear-stress curves in addition of plotting viscosity vs shear-rate. It is remarkable from the Figure 3 that the curve of the sample (S1) is characterised by a sharp, precipitous fall-off from a high-viscosity Newtonian plateau at low shear stress, then an equally sharp levelling-off towards a low-viscosity asymptote. This type of behaviour is associated with the behaviour of very shearthinning slurries. Figure 3 demonstrates that the viscosity decreases when augmenting the shear stress then it captures an asymptotic behavior. It is also noted that the plots $\log$ (viscosity) vs. log (shear stress) of the three samples show non-straight lines; this can be explained by the effect of the particle roughness surface i.e. smooth or sharp. Other artifacts can also be present, even when end effects are eliminated in a well-made viscometer, there can still be wall effects giving real or apparent slip effects. This usually gives a little bit lower-than-expected viscosity. These can be overcome by roughening the surface of the viscometer geometries in contact with the liquid being measured.

Figure 4 shows the adjustment of the rheogram obtained experimentally of the three samples to the rheological models. The rheological models have already been detailed in previous works. We did try to adjust the exper- 
imental data obtained for the three samples. It is found that the model which perfectly describes the rheological behavior the sample (S1) is the Casson model $\sigma^{1 / 2}=$ $20,815 \cdot y^{1 / 2}+47,277$ with an $R^{2}=0,9957$ where $\vartheta=$ $a^{2}$ and the (yield stress) $=\mathrm{b} ; \sigma$ : shear stress. As regards the sample (S2) we note that the Bingham model $\sigma=$ $0,02017 . y+6,9709$ seems to be the perfect model that describes the fluid flow rheological behavior $\left(R^{2}=0,977\right)$ where $\vartheta=a$ and the (yield stress) $=\mathrm{b}$. The Bingham model is a model that defines the behaviors of the slurries having linear rheological profiles. From figure 4 we notice that the Bingham model suits perfectly the rheological behavior of sample (S3). The viscosity and yield stress values for the Bingham model are: $\vartheta=a=0,0184 \mathrm{~Pa} \cdot \mathrm{s}$ and yield stress $\mathrm{b}=0,0865 \mathrm{~Pa}$.

\subsection{Small-Angle X-ray Scattering}

All three powders showed power law behaviors with only very little deviation from the investigated q range (0.01$\left.0.38 \AA^{-1}\right)$. The modulus of $q$, the scattering vector, is represented by the following formula in terms of the wavelength of the X-rays $\lambda=1.54 \AA$ and the scattering angle between the incident X-ray beam and the detector measuring the scattered intensity $2 \theta$ :

$$
q=(4 \pi / \lambda) \sin (\theta)
$$

The samples were fitted with the Fisher-Burford fractal model. It is a power-law model with a cross-over to a low-q Guinier region and a constant background of the form:

$$
I(q)=A \cdot\left(1+q^{2} \frac{2 R_{g}^{2}}{3 \alpha}\right)^{-\frac{\alpha}{2}}+B
$$

Where $I(q)$ is the intensity, $A$ is the overall scale factor, $B$ the constant background, $R_{g}$ the Guinier radius of gyration, and $\alpha$ the surface fractal dimension [13].

Figure 5 shows Porod plots resulting from the desmeared SAXS data of the three powder dust samples. The data follow approximately $q^{-3.8}$ to $q^{-3.9}$ for all three powders indicating a fractal surface structure of quite large powder particles with a rough surface interface. Therefore the associated surface-fractal dimension are of 2.2 for the sample S1 and 2.1 for both samples S1 and S2.

SAXS data of the sample S1 was fitted with a pure power-law expression without the cross-over to a low-q Guinier region as shown in Figure 5 (A) thus $R_{g}$ could not be precisely determined because of the large structures present in the sample $\mathrm{S} 1$ that necessitating data to smaller
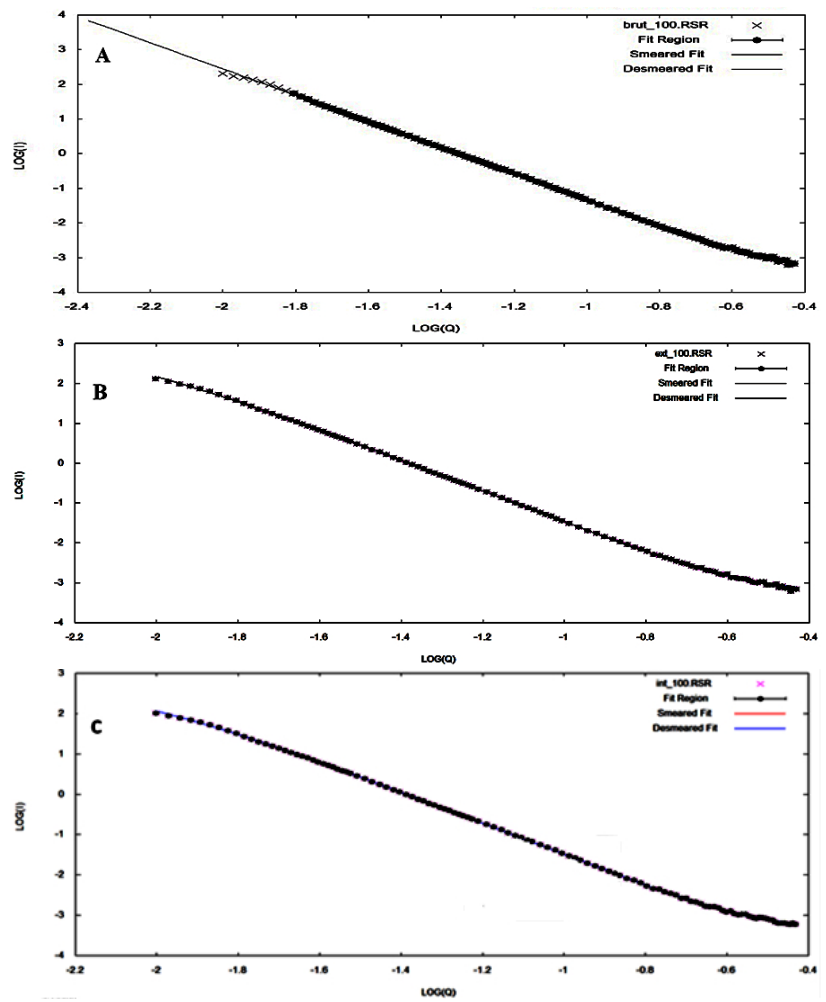

Figure 5: Small-angle-X-ray scattering profiles of the three samples. Data plotted as a log (I (q)) vs. $\log (\mathrm{q})$. (A) sample S1: untreated phosphate dust. (B) sample $S 2$ : calcium phosphate dust from the outside of filter. (C) sample S3: calcium phosphate dust from the inside of filter

q-values than what is available in the experiment. Guinier analysis of the $\mathrm{S} 2$ and $\mathrm{S} 3$ samples profile yields a radius of gyration of $99.72 \pm 2.87$ and $83.87 \pm 1.93 \mathrm{~nm}$, respectively. The radius of gyration $R_{g}$ derives the information concerning the overall particle size. The $R_{g}$ is the root-mean-square distance from either the center of density or a given axis to the molecule weighted. The smaller structure characterized by $R_{g}=83.87 \pm 1.93$ are attributed to the sample S3 obtained from the inside of the filter bag. These phosphates dust are responsible for the phenomena of clogging in depth.

Figure 6 (A) is a data plotted of the three samples as $\log (I(s))$ versus $\log (s)$. The scattering profiles for the different samples are very much alike, which designates that their nanostructures are very close in terms of size and shape of the particles. The scattering curves of the dust phosphate samples revealed that the average Porod slope in the $q$ ranges was -4 . A slope of -4 characterize rough interface of fractal dimension. Additionally, particles are with all sizes and shapes and too big to be resolved.

Figure 6 (B) displays Porod plots which present relation of $I(s)^{\star} s^{4}$ versus s. Some oscillations appear in the pro- 


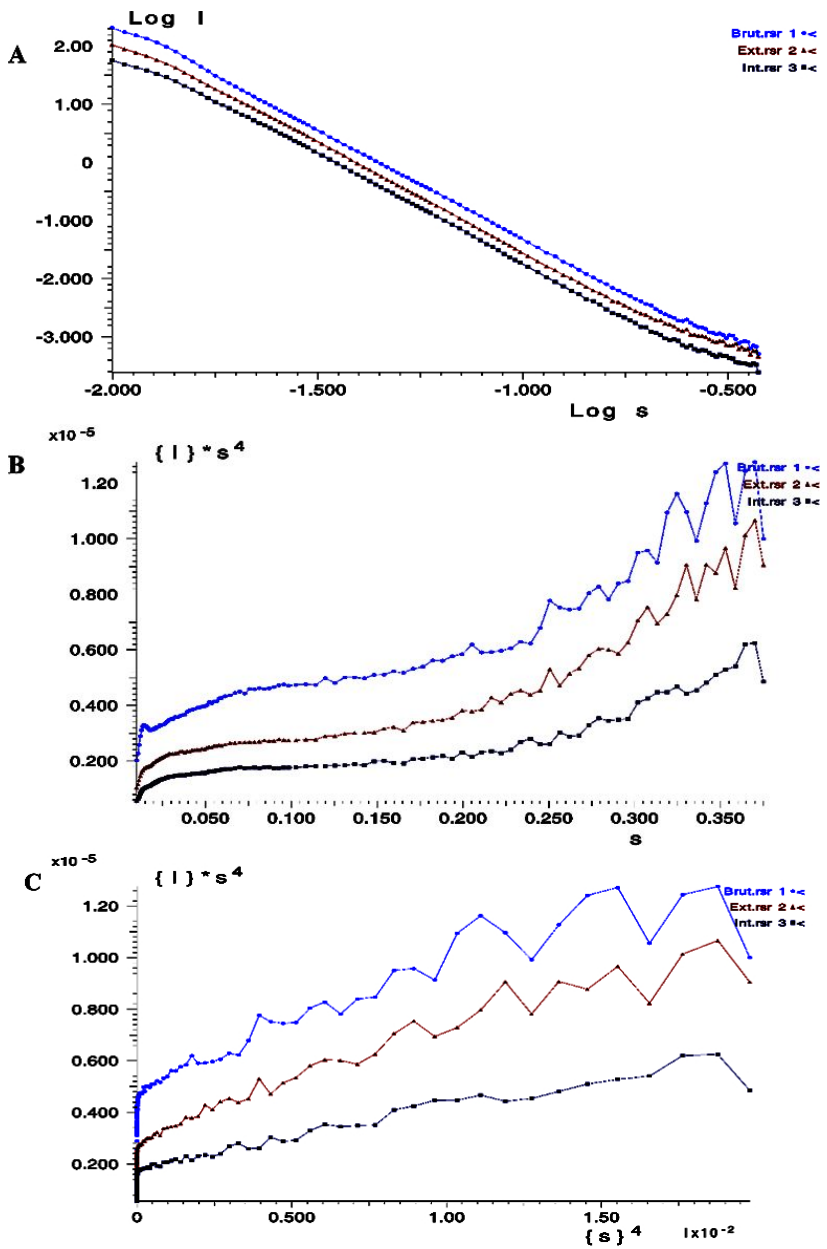

Figure 6: Small-angle-X-ray scattering profiles of the three samples. (A): Data plotted as a $\log (I(s))$ vs $\log (s)$. (B): Porod plot to show $q^{-4}$ dependence behavior $\left(I(s) \times s^{4}\right.$ vs. s). (C): Plot of $I(s) \times s^{4}$ vs. $\left.s^{4}\right)$. Sample S1: untreated phosphate dust (blue). Sample S2: calcium phosphate dust from the outside of filter (red), and sample S3: calcium phosphate dust from the inside of filter (purple)

files of the samples S1 and S2, revealing that the phosphate particles are less polydisperse than in the sample S3. For a polydispersion the radius of gyration is a biased average which largely overvalues the contribution of the lager particles. The power law $\mathrm{s}^{-4}$ expects that the particles are of a spherical shape tending to be globular. The Porod-Debye region defines for a compact, folded particle a scattering intensity decay related to s-4 [14]. Figure 6 (C) illustrates the behavior of $\mathrm{I}(\mathrm{s})$ at high $\mathrm{s}$ which was analyzed using a Porod-Debye plot $\left(I(s) s^{4}\right.$ versus $\left.s^{4}\right)$ that is expected to be asymptotically constant. The plots shows curves with important shape-dependent oscillatory features, particularly for the samples S1 and S2. This plot is exposed here as the asymptotic tendency of $I(s) \mathrm{s}^{4}$ to a constant that is implied by the Porod law. The ratio of surface area to vol- ume and concentration are proportional to the asymptote amplitude.

\section{Conclusion}

The rheological characterization of the dust phosphates deposits obtained from the baghouse dust collector showed that the pseudoplastic behavior of untreated dust phosphate changes to Bingham behavior during filtration/cleaning process. Additionally, the viscosity decreased throughout the drying process. SAXS analysis presented in the preceding section clearly demonstrate that the structure of the phosphate dust is spherical with a radius of gyration in the range from $80 \mathrm{~nm}$ to $100 \mathrm{~nm}$ for the two samples S2 and S3 withdrawn from the baghouse dust collector meanwhile for the untreated phosphate dust, it was difficult to determine the gyration radius because of the large particles containing in the sample. Concerning the surface characteristics, it was concluded that for all samples there was a fractal surface structure of quite large powder particles with a rough surface interface.

In this paper we described the local structure of the dust samples and the determination of rheological parameters. This work demonstrated the dependence of the dust characteristics on the filtration process.

Acknowledgement: Special thanks to Prof. Dr. Jan Skov Pedersen and Jeppe Lyngsø from Aarhus University Denmark for the SAXS apparatus.

\section{References}

[1] Andrew B., Dust control handbook for industrial minerals mining and processing. U.S. Department of Health and Human Services, Public Health Service, Centers for Disease Control and Prevention, National Institute for Occupational Safety and Health, 2012.

[2] Milosevski M., Bossert J., Milosevski D., Gruevska N., Preparation and properties of dense and porous calcium phosphate, Ceram. Int., 1999, 25, 693-6.

[3] Thangamani N., Chinnakali K., Gnanam F., The effect of powder processing on densification, microstructure and mechanical properties of hydroxyapatite, Ceram. Int., 2002, 28, 355-62.

[4] Pattanayak D.K., Dash R., Prasad R.C., Rao B.T., Rama Mohan TR., Synthesis and sintered properties evaluation of calcium phosphate ceramics, MAT. SCI. ENG. C., 2007, 27, 684-90.

[5] Raynaud S., Champion E., Lafon J., Bernache-Assollant D., Calcium phosphate apatites with variable $\mathrm{Ca} / \mathrm{P}$ atomic ratio III. Mechanical properties and degradation in solution of hot pressed ceramics, Biomaterials, 2002, 23, 1081-9. 
[6] Adalja S. B., Otaigbe J. U., Melt rheology of tin phosphate glasses. Appl. Rheol., 2001, 11, 10-18.

[7] Shi F.N., Napier-Munn T.J., A model for slurry rheology, 1996, 21.

[8] Prélot B., Zemb T., Calcium phosphate precipitation in catanionic templates. MAT. SCI. ENG. C., 2005, 25, 553-9.

[9] Matsumoto T., Chiba J., Rheological and small-angle X-ray scattering investigations on the shape and ordered arrangement of native ovalbumin molecules in aqueous colloids, J. Chem. Soc. Faraday Trans., 1990, 86, 2877.

[10] Matsumoto T., Inoue H., Small angle X-ray scattering and viscoelastic studies of the molecular shape and colloidal structure of bovine and rat serum albumins in aqueous systems, J. Chem. Phys., 1993, 178, 591-8.

[11] Pedersen J.S., A flux- and background-optimized version of the NanoSTAR small-angle X-ray scattering camera for solution scattering, J. Appl. Crystallogr., 2004, 37, 369-80.
[12] Yuan J., Murray H.H., The importance of crystal morphology on the viscosity of concentrated suspensions of kaolins, Appl. Clay Sci., 1997, 12, 209-19.

[13] Bünger M.H, Foss M., Erlacher K., Hovgaard M.B., Chevallier J., Langdahl B., Corrigendum to "Nanostructure of the neurocentral growth plate: Insight from scanning small angle $\mathrm{X}$-ray scattering, atomic force microscopy and scanning electron microscopy", Bone, 2007, 41, 157.

[14] Rambo R.P., Tainer J.A., Super-Resolution in Solution X-Ray Scattering and Its Applications to Structural Systems Biology, ANNU. REV. BIOPHYS., 2013, 42, 415-41. 